\title{
Discussion of Data Mining in Medical Insurance System Ning-Can LONG
}

The First Affiliated Hospital of Jinan University

hqyy2016@163.com

Key words: Medical insurance, Data mining, Data warehouse, Data sharing.

\begin{abstract}
This paper describes some problems in medical insurance management, introduces the advantages of application of data warehouse and data mining technology in the data of medical insurance information system.
\end{abstract}

\section{Introduction}

With the deepening of the medical reform in our country, the scope of medical insurance has been expanded and the level of protection has been improved. The proportion of patients with medical insurance in the hospital is higher and higher, and the proportion of medical insurance settlement income in the hospital's operating income is also growing.

How to manage the health insurance fund to ensure that the majority of insured people's life-saving money can be fully utilized, has become an important work of medical insurance management. As the hospital and health insurance belonging to different management department, there is incomplete exchange of information and asymmetric phenomenon, which will increase the operation risk for the hospital and medical insurance management.

\section{Problems Faced by Medical Insurance Management Institutions and Hospital in Medical Insurance Management}

1) The medical insurance institution is the administrator of the medical insurance system(MIS), and the hospital is the main carrier of the implementation of the MIS. They are belonging to different departments with the phenomenon of incomplete and asymmetric information exchange.

2) How to reasonably control the increase of medical expenses of patients with medical insurance, and provide the perfect medical service for patients with medical insurance.

3) How to make a scientific and reasonable quota of medical insurance.

4) How to set up a scientific method for the management of medical insurance fund, just as fund balance, operation analysis, risk early warning, budget control, etc.

5) How to conveniently and quickly serve the patients with medical insurance to avoid the cumbersome procedures and low efficiency.

6) How to carry out the policy of medical insurance, just like medical insurance, the use of diagnosis and treatment catalogue, the limit of single disease expenses, the patient information inquiry, the expenses control, etc.

In order to solve the problems above, we will use data warehouse (DW) and data mining (DM) technology to filter, integrate and store the historical and current data of MIS and hospital information system(HIS). DW and DM is a new subject for the medical insurance and hospital information, and it has become an inevitable trend of the development of medical insurance and hospital information.

\section{Implementation Methods}

1) Using the technology of data warehouse, data interface table(Table 1), the expenses of the type table, Medicare drug table, diagnostic table, medical insurance patient information table, medical insurance patient order list and Medicare patient expenses schedule etc. can be established for MIS and the HIS. 
Table 1 Data interface table

\begin{tabular}{|l|l|l|l|}
\hline HIS Charge Type & Western Medicine Fee & Western Medicine Fee & $\ldots$ \\
\hline Charge code & 002001IJ0 & 01004 CP0 & $\ldots$ \\
\hline Name of hospital charges & Houttuynia Injection $(100 \mathrm{ml})$ & Amoxicillin Capsule & $\ldots$ \\
\hline Hospital dosage form & Injection & Capsule & $\ldots$ \\
\hline Types of medical insurance & Western Medicine Fee & Western Medicine Fee & $\ldots$ \\
\hline Health insurance code & 1003012 & 1001001 & $\ldots$ \\
\hline Health insurance project & Houttuynia & Amoxicillin Capsule & $\ldots$ \\
\hline Medicare dosage form & Injection & Tablet & $\ldots$ \\
\hline Payment ratio & 1.00 & 1.00 & $\ldots$ \\
\hline
\end{tabular}

2) Creating data interface control module for MIS and HIS. It will ensure that the HIS' drugs, diagnostic table, expenses type, disease directory and the Medicare directory were be controlled and indicated. Doctors can generate data by DM technology, according to the type of patient's Medicare, illness, doctor level, tips or restrictions on the use of drugs, diagnostic and therapeutic attributes.

3) Creating data update module of MIS and HIS, in order to timely renew the MIS and HIS when adding, modifying the patients' information, medicine, diagnosis and treatment, expenses type, disease, etc.

4) Making the hospital-side medical insurance patient details, detailed details of the expenses of real-time transmission module, the medical insurance patient's doctor's orders, fees and other real-time introduction to the health insurance system database for medical insurance management of medical insurance patients in real-time audit, supervision, statistics, analysis to help hospitals implement the health insurance policies.

5) Through the data mining production of hospital-side medical insurance patient expenses query report(Table 2), hospital's departments will real-time query the medical insurance patients hospitalization expenses, the proportion of expenses, reasonable control of medical insurance patients rise in medical expenses to ensure the realization of medical insurance quota indicators.

Table 2 Medicare patient expenses query report

\begin{tabular}{|l|l|l|}
\hline Patient name & Zhang San & $\ldots$ \\
\hline Dept. & Cardiovascular & $\ldots$ \\
\hline Proportion of Group A Western Medicine(\%) & $73 \%$ & $\ldots$ \\
\hline Proportion of Group B Western Medicine(\%) & $23 \%$ & $\ldots$ \\
\hline Proportion of Western Medicine Expenses(\%) & $3 \%$ & $\ldots$ \\
\hline Diagnosis and Treatment Expenses be Reimbursed(\%) & $85 \%$ & $\ldots$ \\
\hline Material Expenses be Reimbursed(\%) & $70 \%$ & $\ldots$ \\
\hline High Value Consumptive Material(\%) & 0 & $\ldots$ \\
\hline Cost of Drugs(\%) & $40 \%$ & $\ldots$ \\
\hline Cost of Medical(\%) & $59 \%$ & $\ldots$ \\
\hline Total Expenses be Reimbursed(\%) & $87 \%$ & $\ldots$ \\
\hline Total Expenses & 6233 & $\ldots$ \\
\hline
\end{tabular}

6) DM for medical insurance institutions can make use of the medical insurance fund has been the use of statements, no payment of the statements, combined with a certain data model to generate budget statements, and to develop scientific and reasonable medical insurance quota indicators to protect the health insurance fund controllable, smooth operation. For example, the medical insurance fund budget through this year and last year reimbursement expenses of patients, patients at hospital expenses, the proportion of the insured, increasing the proportion of patients with the 
disease type in recent years, proportion of CPI growth, the concept of social development and data analysis, formulate scientific and reasonable fund budget.

7) Creating the medical insurance patient settlement module. According to the settlement function provided by the MIS, HIS can set up the corresponding insurance reimbursement settlement module, to avoid the 2 settlement procedures (each of hospital and Medicare reimbursement) and reduce staff workload, improving work efficiency.

8) Using DW \& DM to create Medicare patients' information database and upload \& query module, to upload into the medical library at the provincial level timely. Medical insurance agencies at all levels can query hospitalization Medicare patients conveniently and also check out more fake invoices.

9) Hospital according to the medical insurance settlement funds, from the data analysis of the reasonable allocation of medical insurance return resources, will mobilize the enthusiasm of the department, promote the hospital departments to implement the medical insurance policy.

\section{Advantages of Medical Insurance and Hospital Data Sharing}

1) The application of Medicare data interface control table in HIS can greatly simplify the management of medical insurance patients. Now the hospital uses three categories of Medicare drugs dictionary and dictionary is not completely consistent with the diagnosis and treatment project which will reduce the quality of hospital management of patients with medical insurance.

2) Data update reminder module will enable front-line operators to master the changes in the health care policy and hospital data, in order to better reimbursement for the expenses of medical treatment.

3) The development of medical insurance quota is more scientific and reasonable, and the use of medical insurance fund is more controllable. For example, if the medical expenses of the medical insurance patients are not transmitted in real-time, the medical insurance funds of the unqualified patients are not calculated by the medical insurance fund in the analysis and budget analysis of the regular medical insurance fund. It is very easy to cause the medical insurance fund to use the analysis inaccurate, and the budget won't be reasonable.

4) Medical information sharing of patients will provide facilitate to the hospital management of medical patients, to avoid impostor, to identity information error, to avoid the patient running back and so on. Medical management departments at all levels of medical institutions, including reimbursement departments from the Medicare system will obtain medical information from patients to facilitate the medical insurance management staff whom arrive at the department to check the medical information of patients (some without the medical card or health insurance card information incomplete, etc.), so that patients can appreciate the convenience and advantages of health insurance.

5) Medical insurance agencies can review the medical insurance patients' doctor's orders and fees anytime, and regulate the medical institutions at all levels of medical behavior, meanwhile supervision of "big prescription" and "excessive medical behavior".

6) HIS through the DM can easily query the medical insurance hospital inpatient departments, the proportion of expenses, the distribution of funds, the use of funds, etc.. It will provide a scientific basis for improving implement the health insurance policy.

7) Medicare reimbursement speeding, procedures simplifying. Health insurance system and the HIS connection, will omit the artificial secondary medical expenses and artificial differences medical insurance project work.

8)Reducing the cumbersome tune. Medical insurance patient's doctor's orders, medical records, the expenses of information in a timely manner to facilitate the supervision and management, simplifying the work process, reducing management costs.

With the development of medical insurance and hospital information construction, will produce massive amounts of data, how to find the data we need from these massive databases, deep-level rule extraction and experience, and get useful information on management decisions to meet us of the management requirements, has become a future research direction. 


\section{References}

[1] Huang Jing, Guo Wenming. Data mining research on the management of hospital health care Branch [J]. medical equipment, 2009,30 (3): 53-54.

[2]Chen Wenwei. Data warehouse and data mining [M]. Beijing: Tsinghua University press, 2006

[3] Guo Ying, Kong Nanling, Gao Xin, et al. Take the initiative to undertake social responsibility and improve the management of health care [J]. China health quality management, 2009, (4): 30-32.

[4] Zhang Ying. Discussion on the interface between medical insurance and hospital information management system [J]. medical equipment information, 2006,21 (9): 49-50.

[5] Wang Xuefeng. Research on medical data sharing framework based on IHE [D]. Shanghai Jiao Tong University, 2010

[6] Li Dongwu. On the interface construction of hospital information system and medical insurance information system [J]. medical information, 2008, (3): 317-319.

[7] Zhang Yi, Zhang Guoying, Ma Zongfeng, et al. Standard for realizing the interface between medical insurance system and HIS system [J]. Journal of Chengde Medical College, 2010(3):296-297.

[8] Su Shaosheng, Cheng Minting, Zhang Shujuan. A large hospital to carry out data mining project problems and Countermeasures of [J]. Chinese medical equipment, 2010,25 (8): 59-60.

[9] Han Yu. Application of data mining technology in hospital information system [J]. Journal of medical informatics, 2010, (10): 28-31.

[10] LI Jing, HU Liyong, XIAO Ou. Application of Data Mining Technology in Medical Insurance Management Platform [J]. China Digital Medicine, 2016, 11(5): 79-80.

[11] LI Sheng. Application and Research of Data Mining in Medical Insurance Monitoring in Yili Area [J]. Heilongjiang Science and Technology Information, 2016, (15): 53-54.

[12] DAI Zikai. Research on Data Mining Engine Design in Medical Insurance Audit System [D]. Zhejiang Gongshan University, 2016. 